\title{
MARKETING EXPERIENCIAL PARA LA PROMOCIÓN DEL PROCESO EDUCATIVO A NIVEL DE PREGRADO: UN ESTUDIO DE DOS UNIVERSIDADES PRIVADAS ${ }^{*}$
}

\author{
MIGDALIA CARIDAD ${ }^{* * *}$, MARÍA ISABEL CASTELLANO ${ }^{* * * *}$, MARÍA FERNANDA HERNÁNDEZ ${ }^{* * * * *}$ \\ UNIVERSIDAD DE LA COSTA - CUC
}

Recibido/ Received/ Recebido: 02/10/14 - Aceptado/ Accepted / Aprovado: 19/06/15

\begin{abstract}
Resumen
Considerando que las universidades requieren gestionar estrategias que le permitan ofertar sus actividades, productos académicos, investigativos y de extensión, a fin de no perder competitividad en el mercado, la presente investigación se plantea como objetivo determinar el marketing experiencial para la promoción del proceso educativo a nivel de pregrado en universidades privadas de Barranquilla (Atlántico, Colombia) y de Maracaibo (Estado Zulia, Venezuela). La población estuvo constituida por 300 estudiantes de dos universidades privadas (150 de cada una). El trabajo se sustentó en un estudio cuantitativo y descriptivo. Entre los principales resultados se destaca que el $73 \%$ de los informantes señaló que casi siempre recibe conocimientos de valor, apenas el $10 \%$ experimenta sensaciones agradables en función de sus cinco sentidos al recibir los conocimientos por parte de los facilitadores, y el $37 \%$ indica que siempre los elementos (logo, colores y eslogan) utilizados por la universidad le generan prestigio. Se obtuvo que la base del marketing emocional está en la respuesta positiva que da el cliente al decidirse por comprar el servicio como resultado de una emoción.
\end{abstract}

Palabras clave: Marketing experiencial; Emoción; Proceso educativo.

\section{EXPERIENTIAL MARKETING FOR THE PROMOTION OF THE EDUCATIONAL PROCESS AT THE UNDERGRADUATE LEVEL: A STUDY OF TWO PRIVATE UNIVERSITIES}

\begin{abstract}
Considering colleges require management strategies in order to offer their activities, academic, research and extension products in order to remain competitive in the market, this research therefore
\end{abstract}

\footnotetext{
Este artículo se enmarca en la línea de investigación de Mercadeo, producto del proyecto "Marketing Experiencial para la promoción del proceso educativo a nivel de pregrado en universidades privadas" (2014). Coordinado por la Universidad de la Costa CUC, Barranquilla, Colombia.

** Migdalia Caridad, Lcda. en Comunicación Social por la Universidad del Zulia (LUZ), Venezuela. Magíster en Gerencia de Mercadeo, Doctora en Ciencias Gerenciales y Postdoctorado en Gerencia de las Organizaciones por la Universidad Rafael Belloso Chacín (URBE), Venezuela. Investigadora Adscrita al PEII, categoría B. Docente e Investigadora de la Universidad de la Costa (CUC), vinculada al grupo de investigación de Administración Social de la CUC. Dirección postal: Calle 58 \# 55 - 66. Barranquilla (Colombia). Teléfono: 3362200. Correo electrónico: mcaridad1@cuc.edu.co.

**** María Isabel Castellano, Abogada, Magister en Derecho del Trabajo y Doctora en Ciencias Gerenciales por la Universidad Rafael Belloso Chacín (URBE), Venezuela. Docente y miembro de Comité Académico de la URBE. Correo electrónico: micastellano@urbe.edu.ve.

***** María Fernanda Hernández. Licenciada en Comunicación Social, Magister en Ciencias de la Comunicación y doctorante del Programa Ciencias Gerenciales de la Universidad Rafael Belloso Chacín (URBE). Correo electrónico: maryfermm2014@gmail.com.
} 
seeks to determine experiential marketing for the promotion of the educational process undergraduate level at private universities in Barranquilla (Atlántico, Colombia) and Maracaibo (Zulia State, Venezuela). The population consisted of 300 students from two private universities (150 each). The work was based on a quantitative and descriptive study. The main results highlighted that $73 \%$ of respondents said they almost always receive valuable knowledge, only $10 \%$ experience pleasant feelings based on their five senses when receiving knowledge by the facilitators, and $37 \%$ indicated that elements (logo, colors and slogan) used by the college will always generate prestige. It was found that the emotional marketing is based on the positive response given by the client when he/she decides to buy the service as a result of an emotion.

Keywords: Experiential marketing; Emotion; Educational process.

\title{
MARKETING EXPERIENCIAL PARA A PROMOÇÃO DO PROCESSO EDUCATIVO A NÍVEL DE GRADUAÇÃO: UM ESTUDO DE DUAS UNIVERSIDADES PRIVADAS
}

\begin{abstract}
Resumo
Considerando que as universidades necessitam gerenciar estratégias que lhes permitam oferecer suas atividades, produtos acadêmicos, investigativos e de extensão, a fim de não perder competitividade no mercado, a presente pesquisa se propõe como objetivo determinar o marketing experiencial para a promoção do processo educativo a nível de graduação em universidades privadas de Barranquilla (Atlántico, Colômbia) e de Maracaibo (Estado Zulia, Venezuela). A população esteve constituída por 300 estudantes de duas universidades privadas (150 da cada uma). O trabalho sustentou-se em um estudo quantitativo e descritivo. Entre os principais resultados destaca-se que $73 \%$ dos informantes assinalou que quase sempre recebe conhecimentos de valor; apenas $10 \%$ experimenta sensações agradáveis em função de seus cinco sentidos ao receber os conhecimentos por parte dos facilitadores; e $37 \%$ indica que os elementos utilizados pela universidade (logomarca, cores e slogan) sempre lhe geram prestígio. Obteve-se que a base do marketing emocional está na resposta positiva que dá o cliente ao se decidir por comprar o serviço como resultado de uma emoção.

Palavras chave: Marketing experiencial; Emoção; Processo educativo.
\end{abstract}

Caridad, M., Castellanos, M. \& Hernandez, M. (2015). Marketing experiencial para la promoción del proceso educativo a nivel de pregrado: un estudio de dos universidades privadas En: Revista de la Facultad de Ciencias Económicas de la Universidad Militar Nueva Granada. rev.fac.cienc.econ, XXIII (2), DOI: http://dx.doi.org/10.18359/rfce.1608.

JEL: M31, I21.

\section{Introducción}

Tradicionalmente las empresas $\mathrm{u}$ organizaciones han gestionado el marketing como medio principal para realizar investigaciones de mercado, basándose en que los consumidores llevan a cabo una compra racional de bienes y servicios (productos). Se partía de la premisa que el elemento motivacional principal para adquirirlos es la calidad versus el precio. Actualmente, las propiedades y los beneficios de lo ofertado, pese a ser necesarios, ya no son suficientes para incentivar a una compra, pues la calidad y la innovación en éstos se sobre entiende, y de no contar con los mencionados atributos se torna difícil lograr la cuota representativa en el mercado.

De allí que en la sociedad del siglo XXI la gerencia busca fidelizar a los clientes no solo a través de la transacción, innovación o de las relaciones cercanas, sino que se diseñan estrategias motivacionales emergen- 
tes para captar la atención de nuevos consumidores y mantenerlos en el tiempo que les permita garantizar el éxito de las mismas. Todo ello ha influido directamente en los comportamientos de los mercados del mundo, haciendo necesario el uso del marketing en sus diversas tendencias, incluso utilizando la complementariedad de éstas, para llegar a los diferentes segmentos de clientes de manera certera, aplicable a todas las áreas del quehacer económico-social.

En consecuencia, hay necesidad de ofrecer productos atractivos o satisfactorios y que además tengan un valor adicional basado en aspectos emocionalesvivenciales, ello en la búsqueda de conformar la diferencia con los competidores. Así surge el Marketing Experiencial para dar a conocer la actividad empresarial u organizacional y para generar valor, transmitiendo cercanía, fidelidad, y lealtad en el cliente.

Esta tendencia según Addis (2005) citado en Alcaide (2010), es el conjunto de políticas y estrategias más recientes e innovadoras, centradas en la búsqueda de una nueva fuente de ventaja competitiva basada en la implicación emocional de los clientes y en la creación de experiencias ligadas al producto.

Bajo esta perspectiva, el Marketing Experiencial proporcionaría insumos e inspiración para la promoción de bienes y servicios, entendiéndose que esta última está orientada a efectuar la comunicación entre la empresa y su audiencia objetiva. En este sentido, es oportuno resaltar que la eficacia de la promoción se fundamenta en la utilización del Marketing Experiencial, además, gestionándolo en forma integrada, se convierte en una de las herramientas más utilizadas por los mercadólogos y de las entidades que compiten en el mercado.

Para definir el marketing en forma integrada, los postulados de Belch \& Belch (2005, p. 10) son pertinentes "(...) es el proceso de comunicaciones que implica coordinar los diversos elementos promocionales y otras actividades de marketing que sirven para interactuar con los clientes". En consecuencia la herramienta de la cual se ha venido planteando, ofrece como beneficio principal la interacción directa del público objetivo con la información en general de las empresas u organizaciones.
Rodríguez (2007, p. 61), así lo refiere “(...) un plan de marketing integrado es aquel que proporciona el marco de trabajo adecuado a fin de desarrollar, aplicar y controlar el programa y actividades de comunicación, en este caso, tomando como eje fundacional y rector la creación de una serie de sensaciones nuevas que le aporten algún tipo de vivencia o experiencia al cliente". Ello es consecuencia de la evolución de la disciplina del marketing que en pleno siglo XXI propone centrar la estrategia en el carácter emocional de las decisiones de compra de los consumidores o usuarios y por tanto en la experiencia que despierta una situación de consumo.

Estas premisas igualmente aplican para las universidades. A fin de mantener éstas su prestigio e imagen como responsables de contribuir a la expansión del conocimiento, la divulgación de las ideas, la integración de la cultura, el mejoramiento del sector empresarial, y de la calidad de vida de las comunidades que las rodean; están llamadas a dar visibilidad a sus actividades, presencia, a sus productos académicos, investigativos y de extensión, de lo contrario perderían su competitividad en el mercado ante la oferta educativa de nuevas universidades e instituciones del sector, local o extranjeras, las cuales resultan opciones interesantes para estudiantes que buscan nuevos horizontes.

En dicho proceso es necesario tener en cuenta que los consumidores de una experiencia educativa quieren aprender, ahora bien para lograr ese propósito también quieren hacer, sentir y estar. Bajo este enfoque las universidades tendrían que intentar diseñar experiencias que combinen aspectos relacionados con estos cuatro elementos motivacionales, a fin de lograr experiencias más enriquecedoras y atractivas para el usuario o consumidor. Ante la fuerza de estas premisas surge el interés por desarrollar la presente investigación, la cual tiene como objetivo determinar el Marketing Experiencial para la promoción del proceso educativo a nivel de pregrado en universidades privadas del departamento del Atlántico, Barranquilla, Colombia y del estado Zulia, Maracaibo, Venezuela.

\section{Marketing como creador de experiencias}

Se inicia este aparte marcando la diferencia entre el producto que satisface necesidades y las experien- 
cias que satisfacen deseos. El Marketing Experiencial entiende al consumidor o usuario como persona; mientras el primero compra productos, la persona vive y se emociona, sufre alteraciones de su ánimo, siente interés y participa de lo que está ocurriendo.

Esta reflexión emerge de los planteamientos de Schmitt (2006) quien señala que las experiencias son sucesos privados y se producen como respuesta a una estimulación. Con frecuencia parten de la observación directa y/o la participación en sucesos; se producen como resultado de encontrar, pasar o vivir determinadas situaciones por medio de estímulos que se provocan en los sentidos, en este caso que se conectan a la empresa u organización y la marca con la forma de vida del cliente en un contexto social más amplio.

Otro concepto es el de Lenderman \& Sánchez (2008), se puede afirmar que no difieren del autor citado anteriormente en tanto refieren al Marketing Experiencial como una estrategia que intenta dar vida intencionalmente a la promesa que hace la marca a los consumidores por medio de experiencias organizadas las cuales tienen lugar antes de comprar, durante el proceso de compra y en todas las posibles interacciones posteriores.

Una definición más, Cantero (2013) y Etxebarria (2009), coinciden al indicar que las empresas u organizaciones no gestionan solamente la experiencia de uso del producto gracias a las variables tradicionales del mismo y las características materiales predefinidas, sino que también pueden gestionar el acto de compra y de consumo del producto con el objetivo de agregar emociones y vivencias gratificantes de consumo al valor de la marca, del bien, servicio e ideas.

Esta estrategia en cuestión representa una innovadora herramienta centrada en la implicación emocional y la estimulación de los sentidos y atiende los factores generadores de experiencias tales como sentimientos, pensamientos, acciones y relaciones, factible a ser utilizada por las universidades del sector privado, a fin de marcar la diferencia competitiva en el mercado al momento de gestionar la venta de su producto intelectual. Como expresan Romero, Solórzano \& Gutiérrez (2011) los métodos de instrucción basados en técnicas experienciales tienen el potencial de superar muchas de las limitaciones del paradigma tradicional, pues contienen enfoques diferentes y más complejos, y los resultados permiten la interactividad, promueven la colaboración y el aprendizaje mutuo; permiten abordar aspectos del aprendizaje tanto cognitivos como afectivos, y lo más importante, fomentar el aprendizaje activo.

Retomando el concepto, a fin de evidenciar lo útil de esta herramienta en la educación, para Burgos (2007), el Marketing Experiencial, es aquel que busca incentivar tanto a individuos como a grupos de equipos participantes en programas de incentivos o fidelización para disfrutar actividades únicas. La exclusividad de vivir estas experiencias les da un valor agregado para el público objetivo que se quiere motivar. Lo que viene siendo coherente con la nueva visión del proceso para la formación y aprendizaje, el cual en palabras de Romero, et. al, (2011) parte del principio que las personas aprenden mejor cuando entran en contacto directo con sus propias experiencias y vivencias, más aún si éstas recordaciones son memorables. Es un aprendizaje "haciendo" que reflexiona sobre el mismo "hacer". A propósito de estos principios, resaltan cinco tipos de Marketing Experiencial propuestos por Schmitt (2006), los cuales se mencionan en forma resumida como referencia a los resultados perseguidos:

\subsection{Marketing de sensaciones}

Las experiencias sensoriales se pueden crear a través de la vista, el oído, el tacto, el gusto y el olfato. En el área que ocupa esta investigación, el marketing tendría como objetivo la gestión de la comunicación impactando los cinco sentidos del consumidor, con el fin de influir sobre su comportamiento de compra de conocimientos. Así lo plantea Hormigo (2002) cuando señala que la experiencia sensorial se refiere a como los individuo reaccionan ante un estímulo, en el ofrecimiento de bienes y servicios, ratificando que en la compra y en el proceso de consumo participan los cinco sentidos.

\subsection{Marketing de sentimientos}

Se apela a los sentimientos más internos de los clientes, a fin de crear experiencias afectivas que vayan 
desde estados de ánimo ligeramente positivos vinculados a un producto, hasta fuertes emociones de alegría y orgullo. Lo que se necesita para que el marketing funcione bajo esta estrategia es una comprensión clara de que estímulos pueden provocar tales emociones esperadas.

En relación a los sentimientos, Alfaro (2010) señala que puede referirse a objetos internos (el yo) o externos (la naturaleza); de ahí, la experiencia del sujeto, por muy íntima que sea, le vincula a fuentes personales y a situaciones de su entorno. Son los modos de inserción de los sujetos a la existencia. Otro aporte es la de Moral \& Fernández (2012) cuando señalan que los sentimientos son bloques de información integrada, síntesis de datos de experiencias anteriores, de deseos y proyectos, del propio sistema de valores y de la realidad.

\subsection{Marketing de pensamientos}

Para crear experiencias cognitivas se apela al intelecto. Los pensamientos demandan la atracción de la reflexión convergente y divergente de los clientes. El marketing tiene la posibilidad de aprovechar $y$ a veces mediar lo que las personas reconsideran viejas suposiciones y expectativas. El pensamiento manipula representaciones mentales de información. Una representación puede adquirir la forma de una palabra, una imagen visual, un sonido o un dato en cualquier otra modalidad sensorial almacenada en la memoria. Schmitt (2006) en cuanto a este punto asegura que el pensamiento transforma una representación específica de información en formas y significados nuevos y diferentes, lo que permite responder preguntas, resolver problemas $y$ alcanzar metas.

Podría decirse que es una de las experiencias más pertinentes para el contexto del presente estudio, actividad mental asociada con el procesamiento, la comprensión, la capacidad para recordar y para comunicar. Los psicólogos cognitivos estudian la actividad mental, incluyendo las formas lógicas y a veces ilógicas como se crean los conceptos, se resuelven problemas, toman decisiones y forman juicios, resultados significativos que operacionaliza el Marketing Experiencial.

\subsection{Marketing de actuaciones}

Fundamentalmente se persigue impactar a experiencias corporales, estilos de vida e interacciones. El marketing aquí busca enriquecer la vida de los clientes ampliando sus experiencias físicas, mostrándoles formas alternativas de hacer las cosas, estando de acuerdo con Kotler \& Armstrong (2009). Esto se entiende porque todo organismo funciona dentro de una estructura de comportamiento, de actividad. Para Alfaro (2012) el hombre descompone la actividad en acciones tácticas, o estructuras de comportamiento, articuladas directamente al motivo o no, pero orientadas por una finalidad intermedia consciente.

\subsection{Marketing de relaciones}

De acuerdo con Schmitt (2006) esta experiencia se extiende más allá de los sentimientos individuales y privados del individuo, es la interacción de éste con su yo ideal y otras personas o culturas. Es clave para alcanzar el máximo conocimiento del cliente, indagar sobre sus necesidades, da insumos útiles para poder satisfacerlas y persuadirle para la compra. Igualmente, permite la promoción en forma más económica y confiable. El marketing sustentado en la relación tiene como objeto maximizar la lealtad, a fin de aumentar el valor de cada cliente a largo plazo, de allí que el indicador de esta acción es su fidelidad a la organización o empresa. Alet (2007), en forma acertada señala, para lograr una experiencia positiva con base en las relaciones, debe estar centrada en el cumplimiento de la oferta del producto y su valor agregado, que han sido adaptadas al consumidor de acuerdo con sus características, necesidades y expectativas.

\subsection{Proveedores de experiencias}

Para la creación de los cinco tipos de experiencia mediante la gestión de marketing, es necesario lo que Schmitt (2006) denomina Proveedores de Experiencias. Son instrumentos tácticos dirigidos a crear vivencias basadas en un conjunto de sensaciones, sentimientos, pensamientos, actuaciones y relaciones, mediante el uso de las comunicaciones tanto internas como externas de la empresa u organización, el desarrollo de la identidad visual y verbal (nom- 
bre, logotipo, símbolos), la presencia del producto, las marcas, los entornos espaciales, los sitios web y medios electrónicos y el propio personal. Se amplía a continuación la información en relación a aquellos instrumentos factibles a ser operacionalizados en el proceso educativo:

\subsubsection{Identidad corporativa}

La identidad corporativa es una estrategia global, pues como bien lo establece Chong (2007), ésta se convierte en un valor que la organización genera desde toda su estructura, manifestaciones, actitudes, comunicaciones, estilo y estética. Más aún, es el conjunto de rasgos y atributos que la definen, algunos de los cuales son visibles y otros no, por tanto, tiene que ser con su ser, su forma de ser (comportamiento), y su esencia.

\subsubsection{Entornos espaciales}

Con frecuencia son la expresión más global y cultural, vienen siendo los valores y comportamientos que están detrás de la organización. Zeithaml \& Bitner (2001) aseguran que el lugar físico puede ser más o menos importante para alcanzar el marketing propuesto y otros objetivos que dependen de ciertos factores. En el marketing las decisiones de cómo deben diseñarse las instalaciones puede enfocarse en las necesidades y preferencias del personal y clientes. Así mismo, el lugar puede organizarse para mantener motivados a los empleados y en consecuencia para mejorar la productividad, el trabajo en equipo, la eficiencia operativa o cualquier objetivo del comportamiento organizacional. El ambiente tiene que estar planeado para atraer, satisfacer y facilitar las actividades del personal que mantendrán las relaciones de la empresa u organización con sus clientes como último fin.

\subsubsection{Personal interno}

García (2008) al referirse al personal de contacto con los clientes expresa que son elemento clave para la entrega de un servicio de excelencia y para el logro de una ventaja competitiva, éstos vinculan de manera estrecha las funciones del marketing operativas $y$ de recursos humanos en un esfuerzo por crear un intercambio afectivo de valor entre la organización y sus clientes. Para Kotler \& Armstrong (2009) es el factor crítico que funciona como el eslabón decisivo para llegar a los clientes, pues como puntualiza Pérez (2012) la mayoría de las compras realizadas a diario, llevan un componente emocional importante, ya lo substancial no son los productos sino las personas, el contacto que se tenga con éstas. Igualmente describe algunas claves para alcanzar dichos objetivos como la creatividad, credibilidad y comunicación empática y efectiva con el cliente.

Todas estas herramientas agregan valor al proceso educativo, tornándolo más atractivo y dinámico, si se adecúan a las diferentes metodologías que se vienen aplicando en la formación y capacitación del estudiante a saber. Romero et. al, (2011) clasifica dicha metodología en: a)tradicionales, tales como clases prácticas, seminarios, tutorías, conferencias y b) alternativas al sistema tradicional, donde se encuentran la enseñanza en pequeños grupos, programada, el sistema personalizado de enseñanza, el método del caso, los juegos de empresa, realización de trabajos y prácticas en empresas e instituciones.

\section{Marketing Experiencial desde una visión holística para la promoción}

Diferentes autores orientados en lograr un enfoque holístico en el desarrollo del Marketing Experiencial, vinculan esta disciplina con el proceso de comunicación de marketing integrados (CMI), que implica coordinar los diversos elementos promocionales y otras actividades de marketing que sirvan para comunicarse con los clientes de la empresa u organización, mirando la integralidad de la persona, mente-cuerpo.

Para Lamb, Hair \& McDaniels (2006) la CMI es un método de coordinar con cuidado todas las actividades de promoción (Publicidad, marketing directo, interactivo, relaciones públicas, promoción de ventas, venta personales, marketing de eventos y otras comunicaciones) para un Producto y asegurar la consistencia de los mensajes en cada punto de contacto donde la compañía se encuentra con el consumidor o usuario.

Bien lo señalan Stanton, Etzael \& Walker (2007, p. 508) “(...) es un proceso estratégico de negocios utili- 
zado para planear, crear, ejecutar y evaluar comunicaciones coordinadas, mensurables y persuasivas con el público interno o externo de una organización".

Ahora bien, el principal objetivo de este proceso comunicacional es conseguir una respuesta del destinario del mensaje que proyecta el emisor. De este propósito básico, se deriva el resto de objetivos de la comunicación e impulsa la actuación de sus diferentes instrumentos: informar, persuadir y recordar, de allí que se hable de comunicación de marketing integrada. En palabras de Ildefonso (2006, p. 327), “(...) los objetivos básicos de la comunicación desde el punto de vista de las empresas son los siguientes: informar, posicionar, modificar conductas, enseñar a usar, reforzar conductas, recordar la existencia, estimular la demanda, despertar el interés".

Esta versión está enfocada desde el interior de la organización y para complementar la misma se tienen los postulados de Stanton, Etzel \& Walker (2007) quienes la presentan desde la respuesta del cliente; los autores señalan que la meta u objetivo de la promoción es poner al prospecto al final o en la etapa de compra, pero en la mayoría de los casos esto no es posible mientras la persona no haya pasado por las etapas tales como conciencia, conocimiento, agrado, preferencia, convicción y compra.

Luna (2007) suma otros beneficios al asegurar que la comunicación integrada favorece la integridad, creatividad, mensajes consistentes y diseccionados, más y mejor uso efectivo de los medios, mayor precisión en las estrategias. Genera un sistema de valores y referencias que exhorta a entrar en una nueva relación con los públicos. Situados en el aula como lugar de encuentro y tomando en préstamo esta visión holística bajo los fundamentos del Marketing Experiencial, la "venta" de conocimientos mediante la combinación de métodos interdisciplinarios, permite potenciar el aprendizaje del alumno de una forma más eficaz al aplicar conocimiento y experiencias emotivas a tareas prácticas (Aidar, 2006), además, se logra desarrollar y marcar habilidades relacionadas con la toma de decisiones y la resolución de problemas, el trabajo en equipo, la negociación y el ejercicio del pensamiento crítico (Blasco, 2000; Escobar \& Lobo, 2006).
En este sentido, como ya lo han planteado Palomero et al. (2010), cualquier práctica educativa está guiada integralmente por una posición teórica, por una determinada visión del hombre, del mundo y de la sociedad. Todo proyecto educativo tiene una función política: transformar la realidad, hacer posible la utopía de un mundo más justo e igualitario, objetivo al que el sistema educativo puede contribuir de diferentes formas: convirtiendo las aulas en lugar para la reflexión y el debate; impulsando relaciones dialógicas y solidarias; seleccionando contenidos que permitan hacer una lectura crítica de la realidad, en escenarios atractivos, emotivos, experienciales que faciliten el aprendizaje.

\section{Metodología}

A fin de determinar el Marketing Experiencial para la promoción del proceso educativo a nivel de pregrado en universidades privadas del departamento del Atlántico, Barranquilla, Colombia y del estado Zulia, Maracaibo, Venezuela, se llevó a efecto esta investigación bajo el paradigma denominado positivista, empleando para ello una metodología cuantitativa. Según Barrera (2008), el enfoque positivista, concede primacía a los hechos ante las ideas, a las ciencias experimentales ante las teorías y los postulados de la filosofía. Al respecto, Chávez (2000) determina que el objetivo de la corriente positivista es manifestar la realidad sin modificarla, observándola y recogiendo datos directamente de ella.

Igualmente, el estudio se sustenta en una investigación de tipo descriptivo de campo, bajo la modalidad de investigación aplicativa, pues se caracteriza un hecho y comportamiento de un grupo de individuos, de acuerdo a los postulados teoréticos de Arias (2006) y Bavaresco (2006). Según la evolución del fenómeno la investigación es de tipo transversal, por llevar un proceso de recolección de datos en un tiempo único. Hernández, et al. (2014), indican que el propósito de este tipo de investigación es describir las variables y analizar su incidencia en interrelación en un momento dado. El presente estudio describe la situación del objeto a investigar de forma puntual y en un momento dado; por lo que no requiere de la observación permanente o repetición de estudios posteriores para corroborar su comportamiento. 
La obtención de los datos de información se dio a través de una encuesta con escala Likert, la cual fue aplicada a participantes de pregrado del último semestre, en programas comunes a dos instituciones con vasta experiencia en el área de interés, las universidades "A" y "B" (se omiten los nombres a solicitud de las unidades de informantes), representadas por 300 estudiantes, a saber 150 por institución educativa. La validación del contenido del instrumento se soportó en la revisión de la literatura y el juicio de experto, y para determinar la confiabilidad se estimó el coeficiente Alfa de Cronbach, cuya aplicación arrojó un índice de 0,86\%, para todos los ítems del cuestionario, considerándose de alta confiabilidad según la escala de Hernández et al.(2014), quedando así demostrada su consistencia interna.

En cuanto al proceso estadístico fue realizado con el SPSS y comprendió el análisis descriptivo de las variables en estudio (Martín, Cabero \& De Paz, 2008), así como las medidas de forma como la asimetría y curtosis que según Pérez-Tejeda (2008) sirven para determinar la manera como los datos se reúnen de acuerdo a la frecuencia con que se encuentran dentro de la información, posibilitando la identificación de las características de la distribución e identificar si los datos se distribuyen de forma uniforme y que grado de concentración presentan los valores alrededor del punto central (media aritmética).

\section{Interpretación y discusión de datos cuantitativos}

El análisis cuantitativo de los resultados que arrojó esta investigación se hizo en función de una matriz general que totalizó la data de las dos universidades objeto de estudio, en virtud de que cada registro por separado no reveló tendencias diferenciadoras; de allí la decisión de integrarlas como un todo organizacional. Éste vendría a ser el primer hallazgo, considerando que aun cuando estas instituciones funcionan en diferentes contextos y culturas (colombo-venezolana), las expectativas y experiencias deseadas por los clientes (estudiantes) del sector educativo son coincidentes. El análisis de la siguiente tabla permite explicar de manera detallada lo aquí expuesto:

Tabla 1. Marketing Experiencial

\begin{tabular}{|c|c|c|c|c|c|c|c|c|c|c|c|c|}
\hline \multirow[b]{2}{*}{ REACTIVOS } & \multicolumn{10}{|c|}{ Estadisticos Descriptivos } & \multicolumn{2}{|c|}{ Medidas de Forma } \\
\hline & SIEMPRE & $\%$ & $\begin{array}{l}\text { CASI } \\
\text { SIEMPRE }\end{array}$ & $\%$ & $\begin{array}{l}\text { ALGUNAS } \\
\text { VECES }\end{array}$ & $\%$ & $\begin{array}{l}\text { CASI } \\
\text { NUNCA }\end{array}$ & $\%$ & NUNCA & $\%$ & CURTOSIS & ASIMETRIA \\
\hline - Reciben conocimientos de valor & 0 & 0,00 & 8 & 26,66 & 22 & 73,34 & 0 & 0,00 & 0 & 0,00 & 0,8 & 1,1 \\
\hline $\begin{array}{l}\text { - Experimentan sensaciones agradables en funciòn de } \\
\text { sus cinco sentidos al recibir los conocimientos }\end{array}$ & 3 & 10,00 & 7 & 23,33 & 20 & 66,67 & 0 & 0,00 & 0 & 0,00 & 0,5 & 1,3 \\
\hline $\begin{array}{l}\text { - Los elementos (logo, colores, eslogan) utilizados por la } \\
\text { universidad generan prestigio }\end{array}$ & 11 & 36,66 & 17 & 56,66 & 0 & 0,00 & 0 & 0,00 & 2 & 6,68 & $-5,9$ & 2,2 \\
\hline $\begin{array}{l}\text { - La atenciòn obtenida por parte de los docentes hace } \\
\text { sentir satisfechos(as) a los estudiantes }\end{array}$ & 0 & 0,00 & 2 & 6,68 & 3 & 10,00 & 10 & 33,32 & 15 & 50,00 & $-0,7$ & $-1,1$ \\
\hline $\begin{array}{l}\text { - La variedad de recursos didàcticos (làminas, videos, } \\
\text { conferencias, exposiciones) hace sentir satisfecho al } \\
\text { estudiante }\end{array}$ & 0 & 0,00 & 12 & 40,00 & 0 & 0,00 & 18 & 60,00 & 0 & 0,00 & $-1,9$ & $-0,4$ \\
\hline $\begin{array}{l}\text { - Los estudiantes se sienten satisfechos por los servi- } \\
\text { cios administrativos ofrecidos por la universidad }\end{array}$ & 6 & 20,00 & 0 & 0,00 & 24 & 80,00 & 0 & 0,00 & 0 & 0,00 & 0.5 & 1.5 \\
\hline $\begin{array}{l}\text { - El concepto que tiene de la universidad lo ha formado } \\
\text { a través de la variedad de experiencias positivas que } \\
\text { ha tenido en ésta }\end{array}$ & 4 & 13,33 & 14 & 46,67 & 12 & 40,00 & 0 & 0,00 & 0 & 0,00 & $-0,7$ & 0,4 \\
\hline $\begin{array}{l}\text { - La universidad brinda a sus estudiantes servicios } \\
\text { adicionales dentro de sus instalaciones (wifi, cursos, } \\
\text { becas) }\end{array}$ & 11 & 36,66 & 12 & 40,00 & 6 & 20,00 & 0 & 0,00 & 1 & 3,34 & 2,2 & 1,1 \\
\hline
\end{tabular}

Fuente: Elaboración propia. 
Es interesante constatar cómo en los resultados de la tabla 1 no se haya considerado en gran margen la opción siempre en relación al reactivo: los estudiantes de su universidad reciben conocimientos de valor, por el contrario alcanzó el 0\%, es decir, los encuestados -infieren las autoras- no perciben el proceso de impartir conocimientos innovadores, pertinentes, vanguardistas, con la responsabilidad exigida al docente; casi siempre alcanzó $27 \%$, y el $73 \%$ algunas veces, estos resultados coinciden con las medidas de distribución calculadas, las cuales indican una curtosis de 0,8 para una curva leptocurtica de alta concentración de datos alrededor de las medidas centrales así como un coeficiente de asimetría positivo de 1,1 para una mayor distribución de valores hacia la derecha de las tendencias centrales (alternativas de respuestas 3 y 4). La información obtenida es contraria a lo planteado por Alet (2007), cuando señala que la organización debe enriquecer su propia oferta, de forma que se perciba como única, y para ello tendrá que convertirse en una "creadora de experiencias" por excelencia. Siendo capaz de involucrar al cliente a nivel emotivo, físico, intelectual e incluso espiritual.

Continúan observándose las tendencias altas y polarizadas en el momento de preguntar si los estudiantes de su universidad experimentan sensaciones agradables en función de sus cinco sentidos (vista, oído, sabor, aroma, tacto) al recibir los conocimientos por parte de los facilitadores, el más alto valor (67\%) lo recibió la opción algunas veces, seguido de casi siempre (23\%) y siempre solo el $10 \%$, los resultados coinciden con las medidas de distribución calculadas, las cuales indican una curtosis de 0,5 para una curva leptocurtica de concentración elevada de datos alrededor de las medidas centrales así como un coeficiente de asimetría positivo de 1,3 para una mayor distribución de valores hacia la derecha de las tendencias centrales (alternativas de respuestas 3, 4 y 5). Lo que representa que el docente no muestra como prioridad el uso de diferentes recursos para captar la atención del participante y hacer del acto de socialización del conocimiento una experiencia significativa para el alumno.

La tabla 1 muestra igualmente resultados interesantes cuando se indaga si los elementos (logo, colores, eslogan) utilizados por la universidad generan pres- tigio. Se observa la tendencia de identidad con las instituciones estudiadas, a pesar de las respuestas anteriores que muestran inconformidad con la metodología utilizada por el docente. El 57\% señala que casi siempre, la opción siempre en un $37 \%$ y solo el $7 \%$ aseguró que nunca, los resultados coinciden con las medidas de distribución calculadas, las cuales indican una curtosis de $-5,9$ para una curva platicurtica de poca concentración de datos alrededor de las medidas centrales así como un coeficiente de asimetría positivo de 2,2 para una mayor distribución de valores hacia la derecha de las tendencias centrales (alternativas de respuestas 4 y 5). Estos resultados podrían experimentar una tendencia a mejorar si el aprendizaje, la manera de pensar, interpretar, analizar, conectarse, interactuar, comunicarse y establecer relaciones con los estudiantes se llevara a efecto a través de ofrecer y estimular vivencias emocionales y racionales positivas, así lo manifiesta Schmitt (2007): el marketing da un nuevo giro, el cliente ya no elige un producto sólo por la ecuación costo-beneficio, sino por la vivencia que ofrece antes de la compra y durante su consumo.

El propósito de la interrogante sobre la atención obtenida por parte de los docentes hace sentir satisfechos(as) a los estudiantes, se refiere al hecho de considerar al participante como persona única, integral, racional-emocional. Un 50\% respondió nunca, el $33 \%$ casi nunca, $10 \%$ algunas veces, $7 \%$ casi siempre, y con preocupación se comprueba que siempre alcanzó el 0\%, los resultados coinciden con las medidas de distribución calculadas, las cuales indican una curtosis de $-0,7$ para una curva platicurtica de poca concentración de datos alrededor de las medidas centrales así como un coeficiente de asimetría negativo de -1,1 para una mayor distribución de valores hacia la izquierda de las tendencias centrales (alternativas de respuestas 1, 2 y 3). Una vez más se visualiza que se continúa con la práctica de la masificación de la educación, como si fuese posible prestar un servicio educativo de calidad sin satisfacer las necesidades de los clientes, sin dar atención personalizada y en consonancia con sus expectativas.

Los resultados arrojados difieren de los planteamientos de Rodríguez (2006) quien refiere que existen diversos elementos que influyen en la ca- 
pacidad de un producto para producir placer, satisfacción: a) Factores puramente emocionales. b) Factores cognoscitivos, relacionados con lo que se conoce, con lo aprendido. c) Factores intelectuales, referidos a la satisfacción lógica ante la comprensión de un producto, la atención, la calidad del producto. d) Factores psico-fisiológicos, relacionados con el placer estético.

La siguiente aseveración observada en la tabla 1 viene siendo la variedad de recursos didácticos (láminas, videos, conferencias, exposiciones) hace sentir satisfecho al estudiante, cuyos resultados obtenidos refuerzan los comentarios dados en el párrafo anterior. El $40 \%$ opinó casi siempre, en contraposición con una gran mayoría representada por el $60 \%$ que manifiesta casi nunca. Caso que debe llamar a la reflexión. Los resultados coinciden con las medidas de distribución calculadas, las cuales indican una curtosis de -1,9 para una curva platicurtica de poca concentración de datos alrededor de las medidas centrales así como un coeficiente de asimetría negativo de -0,4 para una mayor distribución de valores hacia la izquierda de las tendencias centrales (alternativa de respuesta 2)

Otro punto débil identificado en los resultados arrojados es el relacionado con la pregunta sobre si los estudiantes se sienten satisfechos por los servicios administrativos ofrecidos por la universidad. Las respuestas estuvieron polarizadas, algunas veces en un $80 \%$ y siempre $20 \%$, los resultados coinciden con las medidas de distribución calculadas, las cuales indican una curtosis de 0,5 para una curva leptocurtica de alta concentración de datos alrededor de las medidas centrales así como un coeficiente de asimetría positivo de 1,5 para una mayor distribución de valores hacia la derecha de las tendencias centrales (alternativas de respuestas 3 y 5). El porcentaje desfavorece la función administrativa de las universidades, olvidando que se debe promover la capacidad consciente de evaluar lo correcto de lo que no lo es en la gestión de los procesos. Las acciones administrativas dirigidas al cliente tendrían que ser valores sustentables en una organización, producto del comportamiento del personal como ser, como profesional, y consensuadas con las normas, principios de la institución, para ofre- cer un servicio de calidad siendo coherente con los objetivos que sustenta el marketing, Rovira (2007) destaca que uno de estos objetivos es comunicar el mensaje adecuado a la persona adecuada y en el momento adecuado.

En la tabla 1 queda demostrado, que existen ciertas debilidades en las instituciones estudiadas que están posicionadas en la mente del estudiante, cuando opinan sobre si el concepto que tiene de la universidad lo ha formado a través de la variedad de experiencias positivas que ha tenido en ésta, así en un $47 \%$ señala que casi siempre, $40 \%$ algunas veces y apenas un $13 \%$ siempre. los resultados coinciden con las medidas de distribución calculadas, las cuales indican una curtosis de - 0,7 para una curva platicurtica de poca concentración de datos alrededor de las medidas centrales así como un coeficiente de asimetría positivo de 0,4 para una mayor distribución de valores hacia la derecha de las tendencias centrales (alternativas de respuestas 3, 4 y 5). Aquí se demuestra que las universidades han llevado a cabo una labor de marketing para vender sus ofertas académicas, sin embargo al analizar los resultados en forma holística e integral se infiere que la gestión de mercadeo ha sido la tradicional sin considerar las nuevas tendencias como el mercadeo experiencial.

La última interrogante que se visualiza en la tabla 1, la cual indaga sobre si la Universidad brinda a sus estudiantes servicios adicionales dentro de sus instalaciones (Wifi, cursos, becas), logró respuestas con valoraciones equilibradas. Así, casi siempre logró el $40 \%$, siempre el $37 \%$, algunas veces el $20 \%$ y nunca alcanzó el 3\%, los resultados coinciden con las medidas de distribución calculadas, las cuales indican una curtosis de 2,2 para una curva leptocurtica de elevada concentración de datos alrededor de las medidas centrales así como un coeficiente de asimetría negativo de 1,1 para una mayor distribución de valores hacia la derecha de las tendencias centrales (alternativas de respuestas 3,4 y 5). Estas respuestas favorecen la práctica del Marketing Experiencial para lograr satisfacción en forma eficiente, no sólo en el interés de querer saber del estudiante, sino cómo lo hago y cómo me siento durante ese proceso. 


\section{Modelo de protocolo de Marketing Ex- periencial en universidades privadas}

Dada la importancia de tener por parte de las universidades privadas una participación óptima en el mercado, y basados en la teoría consultada y datos inferenciales arrojados por esta investigación, se diseñó una aproximación teórica de un Modelo de Protocolo de Comunicación de Marketing Experiencial con el ánimo de crear vivencias positivas en los clientes, ello con el interés de cumplir los objetivos y las metas establecidas por las referidas instituciones. Son cinco elementos manejados en mercadeo, que interactúan en forma interdependiente representados en la gráfica 1. Estos elementos vendrían a ser el cliente, producto, fuerza de venta, técnica de venta y mezcla de medios, los cuales se explican a continuación:

Cliente: Estudiantes. Es fundacional tomar en cuenta sus preferencias, deseos, preocupaciones, necesidades, expectativas, nivel cognitivo. Condiciones que deberían ser, principalmente, conocidos y considerados a la hora de establecer las políticas y estrategias de la institución. Aquí requiere apoyarse en técnicas similares a las utilizadas en la investigación de mercados de marketing como encuestas, paneles, reuniones de grupo y así establecer acuerdos que involucren al estudiante con la gestión de la universidad.

Debido a que los clientes comparan sus percepciones del servicio con sus puntos de referencia cuando evalúan la calidad en el servicio, resulta básico que los profesionales del marketing logren indagar a fondo sobre las expectativas que tienen los consumidores acerca del mismo.

Igualmente, se debe considerar las dos dimensiones de las expectativas: una estructural y otra emocional. La primera se refiere a todo lo asociado con los elementos tangibles del proceso de prestación de un servicio, en este caso las dinámicas de enseñanza (demostraciones, láminas de apoyo, talleres, jornadas, conferencias, videos temáticos, actividades extramuros). La segunda, tiene que ver con el plano emocional del cliente, (estudiante), sobre como espera sentir la experiencia del servicio, aquí es donde se marca la diferencia, es decir, combinado con lo anterior, hay que generar un producto apelando a las sensaciones, percepciones, sentimientos y actuaciones en línea con la teoría de Schmitt (2006).

Ahora bien, en este proceso el producto base esperado sería objeto de atención sin olvidar los complementarios, estos últimos son secundarios pero su importancia puede ser decisiva cuando los resultados competitivos entre productos se equilibran. También es útil considerar los postulados de Rico \& Doria (2003), afirman que a veces hay que sacrificar y perder en corto plazo, generando una experiencia gratificante, para ganar luego en las experiencias brindadas a largo plazo; el desafío de sostener la experiencia del cliente en esta ventana de tiempo, implica sistemáticamente comprender lo que ellos esperan y valoran.

Producto: El producto a ofrecer a este cliente es el conocimiento, el estado de su arte en las diferentes disciplinas que integran la oferta académica, conocimientos emergentes, actualizados, innovadores que fortalezcan las competencias de los egresados. Se ubican aquí también las diferentes estrategias didácticas para vincular al conocimiento en el proceso de la búsqueda del mejor resultado.

Ya en los apartes anteriores se hizo referencia que los productos satisfacen necesidades mientras que las experiencias satisfacen deseos. Se plantea una evolución en la concepción del producto a lo largo del tiempo: bienes, servicios y experiencias.

Este tipo de experiencia que se produce mediante el producto, puede ser directa, cuando el cliente entra en contacto físico con el mismo, lo que ocurre habitualmente en la educación, sin embargo con el uso de la tecnología en el aula también se da la indirecta, cuando es presentando el conocimiento, por ejemplo, virtualmente. En ambos casos el producto es el protagonista de crear sensaciones y satisfacer necesidades en el consumidor.

Es de destacar que uno de los elementos importantes para la generación de experiencias a través del producto es su diseño, colores, formas, estilo, aspectos materiales; el cliente desea obtenerlo no solo porque sea mejor, sino por el significado que 
tiene para él, ya sea porque le ayuda a sentirse satisfecho, o refuerza su identidad como individuo perteneciente a un determinado grupo social o cubrirá sus expectativas en el ámbito profesional. De allí que los conocimientos requieren ser presentados en el aula académica de manera atractiva, que se conecten con las vivencias del estudiante, que le proporcionen beneficios funcionales y simbólicos cobrando especial relevancia los valores sensitivos y emocionales.

Técnica de Venta: Vendría a ser la comunicación personalizada, portadora de un mensaje convincente, fundamentado en el saber y apoyada en el mix de herramientas de marketing. Todo plan de mercadotecnia se basa en la comunicación, necesaria tanto para el éxito como para el logro de los objetivos. Por ende, no se podría hablar de Marketing Experiencial en las universidades sin relacionarlo con la comunicación para la transmisión de conocimientos, para lo cual se necesita establecer un plan académico que permita hacer llegar el mensaje en forma atractiva, dinámica, de calidad, y una vez más se puntualiza, como una experiencia positiva.

En el contexto de esta investigación se requiere del tipo de comunicación interpersonal, relacional. De acuerdo con Schmitt (2006), aplicar el Marketing Experiencial lleva implícito una gran diferencia, reconoce que las personas son únicas, irrepetibles, singulares, reflexivas, inconmensurables y con capacidad de elección, por nombrarse unas. De allí, con su aplicación se evita que al estudiante se le trate de manera impersonal, el tipo de comunicación exigida implica compartir parte de la individualidad y estar consciente de la individualidad del otro.

Igualmente, el docente necesita poseer identidad corporativa, la cual en el punto de venta (aula de clase) se proyecta de forma más completa que cuando se aplica sobre el packaging (embalaje) de un producto, es decir, va mucho más allá de sus impactos visuales, de la exposición del logo, la gráfica y el color, la identidad corporativa envuelve a la persona en una nube de mensajes dirigidos a todos los sentidos -vista, olfato, gusto, tacto y oído con el objetivo de despertarlos, sumarlos y almacenar el impacto en el cerebro (Callís, 2008).
Fuerza de Ventas: Equipo Directivo, todo el personal administrativo de la institución y especialmente los docentes por ser el personal que permanece en contacto personalizado con el cliente (estudiantes), representan la fuerza de venta, éstos últimos, los docentes, deben convertirse en los vendedores de los ideales y de los objetivos de la institución con miras a promover la participación, fidelización de cada uno de los estudiantes. Los clientes quieren disfrutar en los momentos de verdad con el producto, quieren divertirse y emocionarse, para conseguirlo las instituciones necesitan personal motivado que transmita actitud positiva en el contacto con ellos, sumado por supuesto a la efectividad en las resoluciones y la autonomía en las acciones que desempeñen.

En este proceso también hay que resaltar el uso de la comunicación como herramienta para lograr los objetivos del docente, pues las experiencias y calidad de vida están muy relacionadas con las interacciones comunicativas. De allí que la clave no está en el precio, ni en el producto en sí, sino en saber sorprender a través de una estrategia creativa adecuada al público objetivo, saber sorprender positivamente y comunicarla efectivamente (Pérez, 2012).

Mix de Medios: Consiste en el uso conjunto de herramientas de publicidad, promoción de ventas, relaciones públicas, venta personal y marketing directo para promocionar bienes o servicios. Weitz, Castleberry \& Tanner (2005) afirman que los principales elementos de la mezcla de comunicación son la publicidad, las promociones de ventas y la venta personal. Además de informar, persuadir o recordar acerca de los productos, la publicidad se utiliza para crear imágenes de marca, vendría a ser una característica fundamental y muy especialmente para aquellas instituciones que no tienen una clara diferenciación de los atributos de su oferta con respecto al de la competencia. La propaganda y las relaciones públicas forman también parte de las actividades de promoción.

Aun cuando en este proceso la comunicación interpersonal del docente (fuerza de venta), con el estudiante es la que tiene primacía, ésta debe soportarse en la comunicación integral de marketing, donde se suele designar a todos los mensajes planificados por 
las instituciones como apoyo de los objetivos mercadológicos, y por supuesto impactará a los objetivos corporativos, postura que apoyan autores como Martínez (2005). Entre los objetivos de comunicación esta definir la mezcla de medios más adecuada para lograr estos.

Así mismo, según Abikafer et al. (2012), explican que en contraste con las metodologías analíticas, cuantitativas y verbales del marketing tradicional, los métodos y herramientas de un profesional del Marketing Experiencial son diversos y polifacéticos. En pocas palabras, el Marketing Experiencial no está vinculado a una ideología metodológica; es ecléctico, se usa solamente aquello que sea adecuado para conseguir buenas ideas. Algunos métodos y herramientas pueden ser altamente analíticos y cuantitativos o pueden ser más intuitivos y cualitativos.

Gráfica 1. Modelo de protocolo de marketing experiencial en universidades

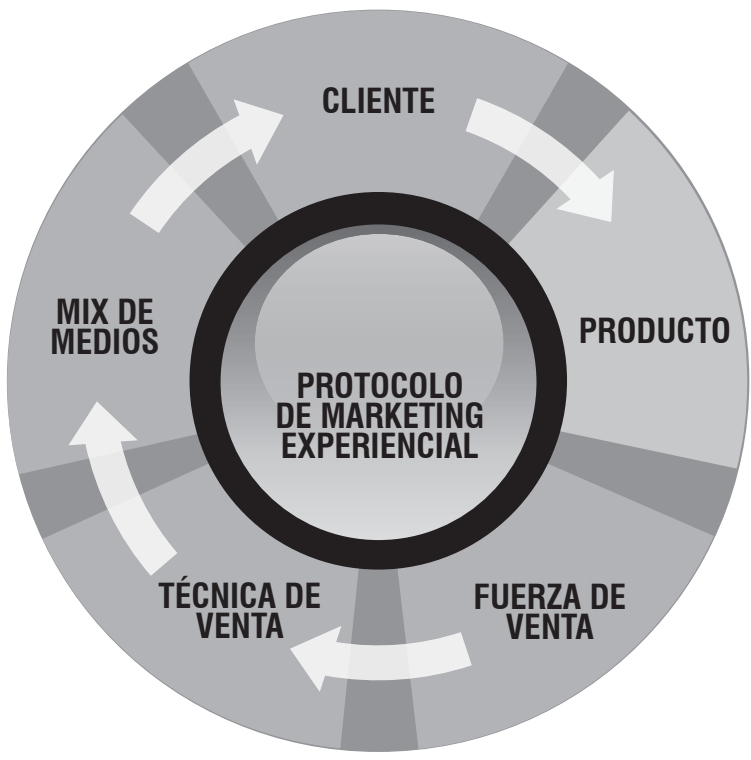

Fuente: Elaboración propia.

En resumen, este modelo intenta orientar hacia una estrategia promocional de Comunicación Integrada apoyada en el Marketing Experiencial, ello traduce que en las instituciones universitarias su servicio básico y complementarios, así como los mensajes corporativos, el posicionamiento, imagen e identidad, tendrían que coordinarse para que las actividades de publicidad, relaciones públicas, presencia institucional y marca, proporcionen un mensaje unificado a través de los distintos canales que se disponen, acciones y estrategias, ello proyectaría la coherencia entre lo que quieren los estudiantes: aprender mediante el hacer, sentir y estar. Siendo esto último la razón de ser del marketing de la experiencia, pues el mismo invita al cliente a vivir sensaciones diferentes y placenteras a partir de los productos. Esta reflexión se sustenta en Francés (2008) quien señala que hoy en día las marcas ya no venden atributos, venden emociones. Asimismo, los productos llamarán a los sentimientos, no a las mentes.

\section{A modo de conclusión}

Esta experiencia que tuvo como objetivo determinar el Marketing Experiencial para la promoción del proceso educativo, es la respuesta al interés de contribuir en que la enseñanza en pregrado no sea una mera transmisión de información sino un proceso de comprensión y asimilación por parte del estudiante, mediante la venta del conocimiento aplicando herramientas del Marketing Experiencial, y así lograr la formación de profesionales competentes en el retador contexto del siglo XXI, quienes a su vez transmitirán sus positivas vivencias que promocionarán las instituciones de su interés.

El estudio permitió, además de verificar el marco teorético contrastado, demostrar que existe la necesidad de contar con la herramienta propuesta: Marketing Experiencial. A la hora de juzgar los métodos utilizados por los docentes, el estudiante consultado, aun cuando reconoce el esfuerzo académico en la facilitación de conocimientos, valora aquellas técnicas integradas en el aprendizaje experiencial que propiciarían al alumno participar activamente en su propio proceso; sería un aprendizaje "haciendo" que reflexiona sobre el mismo "hacer". Esta afirmación se obtuvo en forma coincidente en los estudiantes consultados en ambos países (Colombia-Venezuela), sin presentar diferencias marcadas.

Se observa que en las universidades objeto de estudio no existe mayor fortaleza, en cuanto a la práctica centrada en considerar al participante como persona 
única, integral, racional-emocional. Situación que ayudaría a solventarse mediante la aplicación del Marketing Experiencial, éste parte del principio de atender al cliente (estudiante) como persona única, integral, racional-emocional; el docente no se limitaría a la sola exposición de conceptos (como la lección magistral), sino que a través de la realización de ejercicios, simulaciones o dinámicas, que impacten los cinco sentidos, sensaciones, pensamientos y emociones marcadas por experiencias positivas, la persona asimilaría los principios que le son transmitidos y los ponga en práctica, desarrollando de esta manera un mayor número de competencias personales y profesionales.

Finalmente, se constató que los estudiantes consultados perciben una buena imagen visual de la universidad, no así de la atención ofrecida en sus áreas administrativas. Resultados que demuestran la no integralidad de los esfuerzos de mercadeo en la promoción de estas instituciones, se hace una promesa con la marca que no es cumplida en la práctica, ello con el riesgo de irse deteriorando la imagen posicionada. La atención y operacionalización del marketing de relaciones como parte integral del Marketing Experiencial, prevé no incurrir en dicha falencia.

\section{Referencias}

Abikafer, S. Gonzales, G. \& De la Hoz, R. (2012) Evaluación del Marketing Experiencial aplicado por la empresa Aquaventura Park ubicada en el municipio Maracaibo. Tesis de Grado. Universidad Privada Dr. Rafael Belloso Chacín. Maracaibo, Zulia.

Aidar, A. (2006): "Cases and business games: the perfect match! Developments". En: Business Simluation and Experiential Learning, 33, 204- 212.

Alcaide, J. (2010). Fidelización del Cliente. Editorial ESIC. España

Alet, J. (2007). Marketing Directo e Interactivo: Campañas Efectivas con sus clientes. España: Editorial ESIC.

Alfaro, E. (2012): El ABC del shopping experience. Wolters Kluwer, Vizcaya.

Alfaro, E. (2010): El ABC del customer experience. Wolters Kluwer, Vizcaya.

Arias, F. (2006). El Proyecto de Investigación. Introducción a la Metodología Científica. Caracas.

Barrera, M. (2008). Modelos Epistémicos en Investigación y Educación. (5ta ed.) Caracas, Venezuela: Ediciones Quirón: Fundación SYPAL.

Bavaresco, A. (2006). Proceso Metodológico en la Investigación (Como hacer un diseño de investigación. (5ta ed.). Maracaibo, Venezuela: Editorial de La Universidad del Zulia.
Belch, G. \& Belch, M. (2005). Publicidad y promoción. Perspectiva de la comunicación de marketing integral. México: Mc GrawHill. Sexta edición.

Blasco, F. (2000). "Teoría y aplicación de los métodos de aprendizaje en las Ciencias Empresariales: análisis comparativo entre el Método del Caso y el Juego de Simulación de Empresa”. EU Empresariales, Seminario de Marketing, Madrid, Universidad Complutense de Madrid.

Burgos, E. (2007). Marketing Relacional. Cree un plan de Incentivo Eficaz. Ediciones Netbiblo, S.L. España.

Callís, M. (2008). La ergonomía sensorial en el punto de venta (I). En: Marketing+Ventas, 231, 26-35.

Cantero, J. (2013). "Una definición nada convencional de marketing experiencial: la carta de María a sus Padres". Disponible en: http://marketingexperiencial.wordpress.com/2013/03/05/ una-definicion-nada-convencional-de-marketing-experiencialla-carta-de-maria-a-sus-padres/. Consultado el 16/07/13.

Chávez, N. (2000). Introducción a la Investigación Educativa. ARS Gráfica. Maracaibo, Venezuela.

Chong J. (2007). Promoción de Ventas: Herramienta Básica del Marketing Integral. Argentina: Granica.

Escobar, B. \& Lobo, A. (2006). "Empleo de juegos de simulación empresarial como herramienta para la innovación docente: experiencia en control de gestión de la diplomatura de turismo". En: Revista de enseñanza universitaria, extraordinario, 34- 47.

Etxebarria, J. (2009). "Definiendo el marketing experiencial o emocional”. Disponible en: http://www.komunika.info/artculos/ com-comercial/eventos-ferias-y-plv/definiendo-el-marketingexperiencial-o-emocional/. Consultado el 16/07/13.

Francés, M. (2008). "Marketing Emocional". Disponible en: http:// vivirelmarketing.blogspot.com/2008/05/marketing-emocional. html. Consultado el 16/07/13.

García, K. (2008). Marketing Experiencial: importancia de lograr el contacto y relacionamiento con el cliente. Caso: Falabella. Trabajo de grado para optar al Grado Académico de Master en Administración de Empresas, elaborada en la Universidad Peruana de Ciencias Aplicadas de Lima, Perú.

Hernández, R., Fernández, C. \& Baptista, P. (2014). Metodología de la Investigación. (6a Edición). México. McGraw-Hill. Interamericana Editores.

Hormigo, E. (2002). Dominar el Marketing es Fácil: Revisión de Conceptos de Marketing. España: ESIC Editorial.

Ildefonso, E. (2006). Conducta Real del Consumidor y Marketing Efectivo. España: ESIC Editorial.

Kotler, F. \& Armstrong, G. (2009). Fundamentos de Marketing. México. Editorial Pearson Educación.

Lamb, C., Hair J. \& Mc Daniel, C. (2006), Marketing. (8va Edición). México. Thomson.

Lenderman, M. \& Sánchez, R. (2008). Marketing Experiencial. La Revolución de las Marcas. ESIC Editorial. España.

Luna, P. (2007/Edición Especial) "Marketing integral: comunicación all inclusive". Merca 2.0. México.

Martín, Q., Cabero, M. \& De Paz, Y. (2008). Tratamiento estadístico de datos con SPSS prácticas resueltas y comentadas. Madrid, España: Thomson.

Martínez, I. (2005) "Consecuencias de la Estrategia Integrada de Comunicación". Razón y Palabra, Número 40. Diciembre 2005-Enero 2006. Recuperado el 20 de abril de 2014 de http://www.razonypalabra.org.mx/anteriores/n48/ imartinez.html. 
Moral, M. \& Fernández, M. (2012): "Nuevas tendencias del marketing: El marketing experiencial". En: Revista Entelequia, $\mathrm{N}^{\circ}$ 14, pp: 237-251.

Palomero, J. E., Palomero, P. \& Fernández, M. R. (2010). "El cuaderno de bitácora y la formación de los psicomotricistas. Sobre cómo fomentar el encuentro entre la cultura académica y la cultura experiencial. En: REIFOP, 13(4). Disponible en: http:// www.aufop.com - Consultada en fecha 27/10/205.

Pérez, A. (2012). "Marketing emocional. Una visión desde el coaching". Disponible en: http://www.navactiva.com/es/documentacion/marketing-emocional-una-vision-desde-el-coachingprimera-parte-_58697. Consultado el 16/07/13.

Pérez-Tejeda, H. (2008). Estadística, Ciencias Sociales, del Comportamiento y de la Salud. Cengage Learning Latin America. México.

Rico, R. \& Doria, E. (2003). Retail Marketing, El Nuevo Marketing para el negocio minorista. Editorial Pearson Educations. Argentina. Segunda Edición.

Rodríguez, I. (2007). Estrategias y Técnicas de Comunicación. España: Editorial UOC.
Rodríguez, D. (2006). ¿Cómo mueren los objetos?. Universidad Nacional de Colombia.

Romero, M., Solórzano, M. \& Gutiérrez, M. (2011). "El aprendizaje experiencial como Metodología docente: aplicación del método Macbeth". En: Revista Argos. Universidad Simón Bolívar, 28(54), 127-158.

Rovira, J. (2007). Hoy es Marketing 2007. Editorial ESIC. Madrid.

Schmitt, B. (2006). Experiential Marketing. Cómo conseguir que los clientes se identifiquen en su marca: sensaciones - sentimientos - pensamientos - actuaciones y relaciones. Editorial DEUSTO. España.

Stanton, W., Etzael M. \& Walker B. (2007). Fundamentos de Marketing. 14ta Edición. México: McGraw-Hill.

Weitz B., Castleberry, S. \& Tanner, J. (2005). Ventas. 5ta Edicia Fundamentos de Mrketingblicidad y Marketing. España: McGrón México: McGraw-Hill.

Zeithaml, V. \& Bitner, M. (2001). Marketing de Servicios. Segunda Edición. México: Editorial Mc Graw Hill. 